Research Article

\title{
A New Zoning Method of Blasting Vibration Based on Energy Proportion and Its SVM Classification Models
}

\author{
Haixia Wei, ${ }^{1,2}$ Jinfeng Chen $\mathbb{D}^{1},{ }^{1}$ Jie Zhu, ${ }^{1}$ Xiaolin Yang, ${ }^{1}$ Huaibao Chu, ${ }^{1}$ and Xisen Liu ${ }^{1}$ \\ ${ }^{1}$ School of Civil Engineering, Henan Polytechnic University, Jiaozuo 454000, Henan, China \\ ${ }^{2}$ Fujian Research Center for Tunneling and Urban Underground Space Engineering, Huaqiao University, Xiamen 361021, \\ Fujian, China
}

Correspondence should be addressed to Jinfeng Chen; 2283969514@qq.com

Received 21 October 2020; Revised 19 January 2021; Accepted 1 February 2021; Published 11 February 2021

Academic Editor: Fabio Minghini

Copyright ( $\odot 2021$ Haixia Wei et al. This is an open access article distributed under the Creative Commons Attribution License, which permits unrestricted use, distribution, and reproduction in any medium, provided the original work is properly cited.

The blasting vibration signals show obvious zoning propagation characteristics. Because of not considering the specific influences of geological and topographical conditions, the existing zoning methods of blasting vibration cannot fully describe the internal details of blasting vibration signals. Therefore, a new zoning method of blasting vibration based on energy proportion was proposed in this paper, in which the energy proportion in low, medium, and high frequency bands after multiresolution wavelet analysis is used as the zoning index to distinguish the different characteristics of blasting vibration signals in different zones. Based on the proposed zoning method, 343 sets of measured blasting vibration signals were used to train and test the SVM classification models with four different types of kernel functions. The testing results demonstrate that the zoning method of blasting vibration based on energy proportion has high feasibility, flexibility, and reliability, and the SVM classification models with RBF have higher accuracy than models with other kernel functions in blasting vibration zoning prediction.

\section{Introduction}

Blasting technology not only brings convenience to engineering construction but also causes a series of negative effects, among which blasting vibration effect is the most serious. Under the influences of blasting vibration effect, the buildings around the blasting zone may be damaged and cracked and may even collapse [1-3]. The degree of blasting vibration effect is closely related to characteristics of blasting vibration signals. Therefore, the study on the characteristics of blasting vibration signals is of great theoretical and practical significance for the prediction and control of blasting vibration effect. The peak particle velocity (PPV) is considered to be the most important parameter to represent the characteristics of blasting vibration signals. Many researchers have focused on developing empirical equations and statistical models for estimating PPV [4-8]. In the last few years, artificial intelligent (AI) methods have been widely employed for
PPV prediction, such as artificial neural network (ANN) [9], support vector machine (SVM) [10-12], classification and regression tree (CART) [13], imperialistic competitive algorithm (ICA) [14], genetic algorithm (GA) [15, 16], particle swarm optimization (PSO) $[17,18]$, nested extreme learning machine (Nested-ELM) [19], and so on. In addition to PPV, dominant frequency is also considered as another important index to characterize blasting vibration signals, which has been studied by more and more scholars. Guo et al. [20], Zhou et al. [21], Peng et al. [22], and Gao et al. [23] analyzed the attenuation mechanism and attenuation law of dominant frequency of blasting vibration. Lu et al. [24], Li et al. [25], Liu et al. [26], and Gou et al. [27] proposed the theoretical formulas or prediction models with intelligent algorithms for dominant frequency.

Because of the very complex propagation mechanism and the strong randomness, fuzziness, and uncertainty of blasting vibration, the characteristics of blasting vibration 
signals are quite different in different zones. Some researchers have tried to study the zoning method of blasting vibration and analyze signal characteristics in each zone. Li et al. [28], Pang and Chen [29], and Zhang et al. [30] took the different values of scaled charge or scaled distance as zoning indexes. Meng [31] and Wang [32] selected PPV as the threshold for blasting vibration zoning. The existing zoning indexes such as scaled charge, scaled distance, and PPV only reflect the intensity of blasting vibration signals or its main influence factors including the charge weight and distance from explosion source. Because of not considering the specific influences of geological and topographical conditions, the existing zoning methods of blasting vibration cannot fully describe the detailed characteristics of blasting vibration signals.

Support vector machine (SVM) is a very powerful multifunctional intelligent learning algorithm, which can perform linear or nonlinear regression, classification, and even outlier detection. And the SVM regression algorithm has been effectively applied in the prediction of blasting vibration intensity [10-12]. Because of keeping the original signal details, the time-domain signals can be used for characteristic extraction and classification with the help of appropriate algorithms [33-36]. Compared with other classification algorithms, the SVM classification algorithm has the advantages of relatively small sample demand, low structural risk, and nonlinearity [37], and it has been widely used in various fields [38-40]. Yang and Wu [41] used SVM classification algorithm to discriminate blasting vibration and rock fracture microseismic signals and achieved good classification results. However, how to identify and classify the detailed characteristics of the same blasting vibration signals in different zones remains to be further studied. Therefore, a new blasting vibration zoning method based on energy proportion was proposed in this paper. 343 sets of measured blasting vibration signals were selected for multiresolution wavelet analysis, and the energy distribution characteristics of each subfrequency band were studied. Then, using the proposed zoning method of blasting vibration based on energy proportion, the blasting vibration signals were divided into different blasting vibration zones. Finally, the zoning results were used as samples to train and test the SVM classification models with four different kernel functions. The testing results demonstrate that the new zoning method of blasting vibration based on energy proportion proposed in this paper has high feasibility, flexibility, and reliability, and the SVM classification models with RBF have high prediction accuracy.

The main contributions are given as follows:

(i) A new blasting vibration zoning method based on energy proportion was proposed, in which the energy proportion in low, medium, and high frequency band after multiresolution wavelet analysis is used as the zoning index to distinguish the different characteristics of blasting vibration signals in different zones.

(ii) 343 sets of measured blasting vibration signals were used to train and test the SVM classification models with four different kernel functions based on the proposed zoning method. The comparison results show that the SVM classification models with RBF have higher accuracy than models with other kernel functions in blasting vibration zoning prediction.

(iii) It is proved that the zoning method of blasting vibration based on energy proportion has high feasibility, flexibility, and reliability, and the SVM classification models with RBF have high prediction accuracy. The proposed zoning method and its SVM models can not only provide theoretical guidance for studying the specific characteristics of blasting vibration signals in each zone but also provide an important basis for the prediction, control, and safety standards for blasting vibration.

\section{Classification Principle of SVM Algorithm}

SVM is a machine learning method developed from statistical learning theory, and it has been widely used in solving various classification problems due to its excellent performance [42-45]. The classification principle of SVM algorithm is as follows [46-48].

It is assumed that the training sample set $\left\{\left(x_{i}, y_{i}\right), i=1,2, \ldots, l\right\}$ with size $l$ consists of two categories to keep generality. When $x_{i}$ is the first category, $y_{i}=1$; when $x_{i}$ is the second category, $y_{i}=-1$.

If the sample is linearly separable, there is a classification hyperplane:

$$
w x+b=0 .
$$

Then, make the sample set meet

$$
\begin{cases}w x_{i}+b \geq 1, & y_{i}=1, \\ w x_{i}+b \leq-1, & y_{i}=-1, \\ i & =1,2, \ldots, l,\end{cases}
$$

where $w, x, x_{i} \in R, w, b$, are the parameters to be determined; $w$ is the normal vector of classification hyperplane $w x+b=0$.

The margin between sample point $x_{i}$ and classification hyperplane is defined as

$$
\varepsilon_{i}=y_{i}\left(w x_{i}+b\right)=\left|w x_{i}+b\right|,
$$

where $w$ is normalized and defined as geometric margin:

$$
\delta_{i}=\frac{w x_{i}+b}{\|w\|} .
$$

In order to classify the sample set correctly, it is necessary to select an optimal classification hyperplane among the numerous classification hyperplanes to maximize the distance $\delta$ between the sample set and the classification hyperplane.

When $\varepsilon=\left|w x_{i}+b\right|=1$, the distance between the two types of sample points is $2\left(\left(\left|w x_{i}+b\right|\right) /\|w\|\right)=(2 /\|w\|)$. And the goal is to find the optimal classification hyperplane under the constraint of formula (2) to make $(2 /\|w\|)$ maximum and $\left(\|w\|^{2} / 2\right)$ minimum.

Therefore, the linear separable SVM problem is transformed into a quadratic programming problem: 


$$
\left\{\begin{array}{l}
\min \frac{1}{2}\|w\|^{2} \\
\text { constraint condition: } \quad y_{i}\left(\left(w x_{i}+b\right)\right) \geq 1, \quad i=1,2, \ldots, l
\end{array}\right.
$$

Because the objective function and constraint condition are convex, according to the optimization theory, there is a unique global minimum solution to this problem. Use the Lagrange multiplier method and the KKT (Karush-Kuhn-Tucker) condition:

$$
\alpha_{i}\left(y_{i}\left(\left(x x_{i}\right)+b\right)-1\right)=0 .
$$

The optimal classification function can be obtained:

$$
f(x)=\operatorname{sgn}\left[\sum_{i=1}^{l} \alpha_{i}^{*} y_{i}\left(x x_{i}\right)+b^{*}\right],
$$

TABLE 1: Kernel functions in SVM models.

\begin{tabular}{lc}
\hline Types of kernel functions & Expressions \\
\hline Linear kernel function(LKF) & $K\left(x, x_{i}\right)=x x_{i}$ \\
Polynomial kernel function & $K\left(x, x_{i}\right)=\left(x x_{i}+1\right)^{d}$ \\
(PKF) & $K\left(x, x_{i}\right)=\exp \left(-g \cdot\left\|x-x_{i}\right\|^{2}\right)$ \\
Radial basis function (RBF) & $K\left(x, x_{i}\right)=\tanh \left(k\left(x x_{i}\right)+\theta\right)$ \\
\hline Sigmoid kernel function (SKF) & \\
\hline
\end{tabular}

where sgn is the symbolic function, $a^{*}$ and $b^{*}$ are the parameters to determine the optimal hyperplane partition, and $\left(x x_{i}\right)$ is the dot product of two vectors.

Most problems in engineering practice are nonlinear separable problems, and the quadratic programming problems can be modified as follows by introducing the slack variable $\xi_{i}$ :

$$
\left\{\begin{array}{l}
\min \frac{1}{2}\|w\|^{2}+C \sum_{i} \xi_{i}, \quad \xi_{i} \geq 0, \\
\text { constraint condition: } y_{i}\left(\left(w x_{i}+b\right)\right) \geq 1-\xi_{i}, \quad i=1,2, \ldots, l,
\end{array}\right.
$$

where $\xi_{i}$ is the slack variable and $C$ is the penalty coefficient.

Cortes and Vapnik [49] proposed that the kernel function $K\left(x_{i}, x_{j}\right)$ satisfying Mercer conditions should be used instead of dot product operations to solve duality problems:

$$
K\left(x_{i}, x_{j}\right)=\Phi\left(x_{i}\right) \Phi\left(x_{j}\right)
$$

And the optimal classification function is

$$
\begin{aligned}
f(x) & =\operatorname{sgn}\left(w^{*} \Phi(x)+b^{*}\right)=\operatorname{sgn}\left(\sum_{i=1}^{l} \alpha_{i}^{*} y_{i} \Phi\left(x_{i}\right) \cdot \Phi(x)+b^{*}\right) \\
& =\operatorname{sgn}\left(\sum_{i=1}^{l} \alpha_{i}^{*} y_{i} K\left(x_{i}, x\right)+b^{*}\right) .
\end{aligned}
$$

The kernel functions commonly used in SVM models are shown in Table 1.

\section{Proposal of a New Zoning Method of Blasting Vibration Based on Energy Proportion}

3.1. Principle of Wavelet Analysis. Fourier analysis has solved many problems in signal processing. However, Fourier analysis can only obtain the whole spectrum of the signals, and it has shortcomings in analyzing local characteristics and processing time-varying nonstationary signals. Therefore, wavelet analysis [50], a new time-frequency analysis method, came into being. It retains the advantages of Fourier transform and makes up for the shortcomings in local analysis. Based on different wavelet functions [51], wavelet analysis can accurately reveal the distribution characteristics of signals in time and frequency domain simultaneously.

Meet the condition $[52,53]$

$$
\int_{-\infty}^{+\infty}|\Psi(\omega)|^{2}|\omega|^{-1} \mathrm{~d} \omega<0
$$

where $\Psi(\omega)$ is the frequency-domain representation of the square integrable function $\phi(t)$ and $\phi(t)$ is a basic wavelet or wavelet function.

In the wavelet transform, the basic wavelet function first moves the displacement $\tau$ and then makes the inner product with the signal $X(t)$ in different scales of $a$ :

$$
W T_{x}(a, \tau)=\frac{1}{\sqrt{a}} \int_{-\infty}^{+\infty} x(t) \phi^{*}\left(\frac{t-\tau}{a}\right) \mathrm{d} t, \quad a>0,
$$

where $a$ is the scale factor expanding or contracting with the basic wavelet function $\phi(t)$ and $\tau$ is the response displacement whose value can be positive or negative. Because both $a$ and $\tau$ are continuous variables, the wavelet transform is also called continuous wavelet transform.

The equivalent frequency-domain representation of equation (12) is as follows:

$$
W T_{x}(a, \tau)=\frac{\sqrt{a}}{2 \pi} \int_{-\infty}^{+\infty} X(\omega) \Psi^{*}(a \omega) e^{+j \omega \tau} \mathrm{d} \omega,
$$

where $X(\omega)$ is the frequency-domain representation of $x(t)$.

3.2. Multiresolution Wavelet Analysis of Blasting Vibration Signals. The blasting zone of groundwork excavation project in Rizhao District is about $260 \mathrm{~m}$ long and $120 \mathrm{~m}$ wide, and the excavation depth is $1-6 \mathrm{~m}$. The geomorphic unit of the 
site belongs to the low mountain or hilly area in the Yellow Sea land, and the geomorphic morphological type belongs to the structural denudation residual hill. The bedrock of the site is granite and gneiss, which forms different degrees of weathering zones from top to bottom because of experiencing a long period of geological process. The short-hole expanding blasting method is mainly adopted in the excavation project. The hole diameter is $38 \mathrm{~mm}$, the hole spacing is about $1.2 \mathrm{~m}$, the row spacing is $1 \mathrm{~m}$, and the hole depth is $1.6 \mathrm{~m}$.

In order to control the vibration effect caused by blasting excavation safely, the monitoring work of blasting vibration was carried out in the engineering site during the whole construction process. A large number of blasting vibration signals were measured by IDTS3850 blasting vibration testers and their matching speed sensors. Among them, 343 sets of effective typical signals in the same direction were selected as the sample data of signal identification and zoning in this paper.

The Sadovsky formula is deformed as follows:

$$
v=K\left(\frac{r}{Q^{(1 / 3)}}\right)^{-\alpha},
$$

where $v$ is $\operatorname{PPV}(\mathrm{cm} / \mathrm{s}), K$ and $\alpha$ are the coefficients related to blasting methods and site conditions, $\left(r / Q^{(1 / 3)}\right)$ is the scaled distance $\left(\mathrm{m} / \mathrm{kg}^{1 / 3}\right), r$ is the distance from explosion source $(\mathrm{m})$, and $Q$ is the maximum charge per delay $(\mathrm{kg})$.

The zoning method of blasting vibration based on scaled distance in reference [54] is shown in Table 2.

Using the above zoning method, 343 sets of measured blasting vibration signals were divided into different blasting vibration zones. Then, the multiresolution wavelet analysis was carried out on the 343 sets of signals, and the wavelet basis function $\mathrm{db} 8$ of $\mathrm{dbN}$ wavelet system constructed by Inrid Daubechies was selected [55]. Because the sampling frequency of the blasting vibration testers used in this test is $10000 \mathrm{~Hz}$, according to the sampling theorem, the Nyquist frequency is $10000 / 2=5000 \mathrm{~Hz}$. Since the principle frequency of general buildings is in the range of $2 \sim 10 \mathrm{~Hz}$, the blasting vibration signal at each measuring point is decomposed by wavelet with the scale of 11 , and the corresponding 12 frequency bands are as follows: $0 \sim 2.441 \mathrm{~Hz}, 2.441 \sim 4.882 \mathrm{~Hz}, 4.882 \sim 9.765 \mathrm{~Hz}, 9.765 \sim 19.531 \mathrm{~Hz}$, $19.531 \sim 39.062 \mathrm{~Hz}, \quad 39.062 \sim 78.125 \mathrm{~Hz}, \quad 78.125 \sim 156.25 \mathrm{~Hz}$, $156.25 \sim 312.5 \mathrm{~Hz}, 312.5 \sim 625 \mathrm{~Hz}, 625 \sim 1250 \mathrm{~Hz}, 1250 \sim 2500 \mathrm{~Hz}$, and $2500 \sim 5000 \mathrm{~Hz}$.

Limited by the space, some sets of typical blasting vibration data in the near, medium, and far zones are listed here, as shown in Table 3. The dominant frequency, PPV, and energy proportion results of each subfrequency band of the signals listed in Table 3 by multiresolution wavelet analysis are shown in Tables 4-9.

\subsection{A New Zoning Method of Blasting Vibration Based on} Energy Proportion. It can be seen from Tables 4 to 6 that the zoning method of blasting vibration based on scaled distance basically conforms to the decay law of blasting vibration frequency: the frequency band of blasting vibration signals in the near zone is mainly high frequency band
TABLE 2: Blasting vibration zoning method based on scaled distance.

\begin{tabular}{lc}
\hline$\left(r / Q^{(1 / 3)}\right)$ & Blasting vibration zones \\
\hline$(0,12)$ & Blasting vibration near zone \\
$(12,22)$ & Blasting vibration medium zone \\
$(22,+\infty)$ & Blasting vibration far zone \\
\hline
\end{tabular}

( $\geq 78.125$ ), the frequency band in the medium zone is mainly medium frequency band (9.7657 78.125), and the frequency band in the far zone is mainly low frequency band (0 9.7657). According to the blasting vibration zoning method based on scaled distance, signal 28 in Table 4 is divided into blasting vibration near zone, and the frequency is mainly in the high frequency band. Signal 57 in Table 5 is divided into medium zone, and the frequency is mainly in the medium frequency band. Signal 71 in Table 6 is divided into far zone, and the frequency is mainly in the low frequency band. The above zoning method effectively reflects the frequency decay characteristics of the near, medium, and far zones, which has a certain guiding role in studying the propagation law and characteristics of blasting vibration waves in different zones.

However, the limitations of the blasting vibration zoning method based on scaled distance are obvious. Tables 7 to 9 show the corresponding wavelet analysis results of blasting vibration signals selected separately from near, medium, and far zones based on scaled distance. Through observation, it can be seen that the frequency-band energy proportions of the signals in some blasting vibration zones show the characteristics of other zones, and the examples are as follows. Signal 10 in Table 7 is divided into blasting vibration near zone based on scaled distance; according to the decay law of blasting vibration frequency, the frequency in the near zone should be mainly in the high frequency band, but the dominant energy of the signal is mainly concentrated in the medium frequency band. Signal 6 in Table 8 is divided into blasting vibration medium zone based on scaled distance; according to the decay law of blasting vibration frequency, the frequency in the medium zone should be mainly in the medium frequency band, but the dominant energy of the signal is mainly concentrated in the low frequency band. Signal 5 in Table 9 is divided into blasting vibration far zone based on scaled distance; according to the decay law of blasting vibration frequency, the frequency in the far zone should be mainly in the low frequency band, but the dominant energy of the signal is mainly concentrated in the high frequency band. The existence of these special examples limits the study on the propagation characteristics of the blasting vibration signals in different zones and further affects the control of blasting vibration and the formulation of blasting safety standards.

Therefore, it is more reasonable to take the relative energy proportion of each frequency band as the index to divide blasting vibration zones, and a new zoning method of blasting vibration based on energy proportion was proposed in this paper, as shown in Figure 1.

The zoning method of blasting vibration based on energy proportion can be described as follows: 
TABLe 3: Typical blasting vibration data in the near, medium, and far zones.

\begin{tabular}{lcccccc}
\hline No. & Charge $(\mathrm{kg})$ & Distance $(\mathrm{m})$ & Scaled distance $\left(\mathrm{m} / \mathrm{kg}^{1 / 3}\right)$ & Blasting vibration zone & PPV $(\mathrm{cm} / \mathrm{s})$ & Dominant frequency $(\mathrm{Hz})$ \\
\hline 28 & 6.5 & 15 & 8.0375 & Near zone & 2.3444 & 323.4863 \\
57 & 10 & 36.9 & 17.1275 & Medium zone & 0.8069 & 50.6592 \\
71 & 11 & 63 & 28.3276 & Far zone & 0.0900 & 51.8799 \\
10 & 20 & 26.9 & 9.9100 & Near zone & 0.6891 & 51.8799 \\
6 & 9 & 35 & 16.8264 & Medium zone & 0.5264 & 222.1680 \\
5 & 17.5 & 78.7 & 30.3130 & Far zone & 0.7203 & 42.7246 \\
\hline
\end{tabular}

TABle 4: Dominant frequency, PPV, and energy proportion of each subfrequency band at measuring point 28 in the near zone.

\begin{tabular}{lccc}
\hline Frequency band $(\mathrm{Hz})$ & Dominant frequency $(\mathrm{Hz})$ & PPV $(\mathrm{cm} / \mathrm{s})$ & Energy proportion $(\%)$ \\
\hline $0 \sim 2.4415$ & 1.8 & 0.0454 & 9.5090 \\
$2.4415 \sim 4.8829$ & 3.7 & 0.0134 & 0.1108 \\
$4.8829 \sim 9.7657$ & 6.1 & 0.0287 & 0.2129 \\
$9.7657 \sim 19.5313$ & 12.8 & 0.0325 & 0.1905 \\
$19.5313 \sim 39.0625$ & 28.1 & 0.0430 & 0.1714 \\
$39.0625 \sim 78.125$ & 72 & 0.3041 & 2.9811 \\
$78.125 \sim 156.25$ & 153.8 & 0.3302 & 1.9983 \\
$156.25 \sim 312.5$ & 305.8 & 1.8699 & 64.5484 \\
$312.5 \sim 625$ & 331.4 & 1.2202 & 19.7658 \\
$625 \sim 1250$ & 919.2 & 0.2458 & 0.4337 \\
$1250 \sim 2500$ & 1256.7 & 0.0841 & 0.0740 \\
$2500 \sim 5000$ & 2774 & 0.0300 & 0.0040 \\
\hline
\end{tabular}

TABle 5: Dominant frequency, PPV, and energy proportion of each subfrequency band at measuring point 57 in the medium zone.

\begin{tabular}{lccc}
\hline Frequency band $(\mathrm{Hz})$ & Dominant frequency $(\mathrm{Hz})$ & PPV $(\mathrm{cm} / \mathrm{s})$ & Energy proportion $(\%)$ \\
\hline $0 \sim 2.4415$ & 0.6 & 0.0036 & 0.2496 \\
$2.4415 \sim 4.8829$ & 4.3 & 0.0004 & 0.0005 \\
$4.8829 \sim 9.7657$ & 7.3 & 0.0010 & 0.0016 \\
$9.7657 \sim 19.5313$ & 15.3 & 0.0208 & 0.2916 \\
$19.5313 \sim 39.0625$ & 38.5 & 0.2108 & 17.0179 \\
$39.0625 \sim 78.125$ & 50.7 & 0.5771 & 79.6341 \\
$78.125 \sim 156.25$ & 110.5 & 0.1483 & 2.5940 \\
$156.25 \sim 312.5$ & 177.6 & 0.0358 & 0.1868 \\
$312.5 \sim 625$ & 332.6 & 0.0114 & 0.0160 \\
$625 \sim 1250$ & 748.3 & 0.0027 & 0.0029 \\
$1250 \sim 2500$ & 2150.9 & 0.0026 & 0.0026 \\
$2500 \sim 5000$ & 3251.3 & 0.0021 & 0.0024 \\
\hline
\end{tabular}

Table 6: Dominant frequency, PPV, and energy proportion of each subfrequency band at measuring point 71 in the far zone.

\begin{tabular}{lccc}
\hline Frequency band $(\mathrm{Hz})$ & Dominant frequency $(\mathrm{Hz})$ & PPV $(\mathrm{cm} / \mathrm{s})$ & Energy proportion $(\%)$ \\
\hline $0 \sim 2.4415$ & 0.6 & 0.0453 & 97.8809 \\
$2.4415 \sim 4.8829$ & 4.3 & 0.0005 & 0.0013 \\
$4.8829 \sim 9.7657$ & 9.2 & 0.0005 & 0.0015 \\
$9.7657 \sim 19.5313$ & 17.1 & 0.0043 & 0.0404 \\
$19.5313 \sim 39.0625$ & 29.9 & 0.0224 & 0.5545 \\
$39.0625 \sim 78.125$ & 51.3 & 0.0355 & 1.0447 \\
$78.125 \sim 156.25$ & 80 & 0.0213 & 0.1552 \\
$156.25 \sim 312.5$ & 235 & 0.0499 & 0.2468 \\
$312.5 \sim 625$ & 355.2 & 0.0227 & 0.0561 \\
$625 \sim 1250$ & 943 & 0.0079 & 0.0061 \\
$1250 \sim 2500$ & 1761.5 & 0.0147 & 0.0071 \\
$2500 \sim 5000$ & 2505.5 & 0.0176 & 0.0054 \\
\hline
\end{tabular}


TABLE 7: Dominant frequency, PPV, and energy proportion of each subfrequency band at measuring point 10 in the near zone.

\begin{tabular}{lccc}
\hline Frequency band $(\mathrm{Hz})$ & Dominant frequency $(\mathrm{Hz})$ & PPV $(\mathrm{cm} / \mathrm{s})$ & Energy proportion $(\%)$ \\
\hline $0 \sim 2.4415$ & 2.4 & 0.0040 & 0.2963 \\
$2.4415 \sim 4.8829$ & 2.4 & 0.0023 & 0.0132 \\
$4.8829 \sim 9.7657$ & 8.5 & 0.0025 & 0.0242 \\
$9.7657 \sim 19.5313$ & 17.7 & 0.0755 & 4.8651 \\
$19.5313 \sim 39.0625$ & 36.6 & 0.2197 & 25.3723 \\
$39.0625 \sim 78.125$ & 51.9 & 0.5305 & 66.5348 \\
$78.125 \sim 156.25$ & 94.6 & 0.1026 & 2.8272 \\
$156.25 \sim 312.5$ & 210.0 & 0.0130 & 0.0323 \\
$312.5 \sim 625$ & 360.7 & 0.0060 & 0.0123 \\
$625 \sim 1250$ & 731.2 & 0.0023 & 0.0090 \\
$1250 \sim 2500$ & 1907.3 & 0.0025 & 0.0073 \\
$2500 \sim 5000$ & 3092.7 & 0.0026 & 0.0059 \\
\hline
\end{tabular}

TABLE 8: Dominant frequency, PPV, and energy proportion of each subfrequency band at measuring point 6 in the medium zone.

\begin{tabular}{lccc}
\hline $\begin{array}{l}\text { Frequency band } \\
(\mathrm{Hz})\end{array}$ & $\begin{array}{c}\text { Dominant } \\
\text { frequency }(\mathrm{Hz})\end{array}$ & $\begin{array}{c}\text { PPV } \\
(\mathrm{cm} / \mathrm{s})\end{array}$ & $\begin{array}{c}\text { Energy } \\
\text { proportion }(\%)\end{array}$ \\
\hline $0 \sim 2.4415$ & 1.2 & 0.0408 & 70.2143 \\
$2.4415 \sim 4.8829$ & 3.7 & 0.0012 & 0.0072 \\
$4.8829 \sim 9.7657$ & 7.9 & 0.0026 & 0.0153 \\
$9.7657 \sim 19.5313$ & 14.0 & 0.0045 & 0.0193 \\
$19.5313 \sim 39.0625$ & 25.0 & 0.0125 & 0.1006 \\
$39.0625 \sim 78.125$ & 53.7 & 0.0780 & 1.5408 \\
$78.125 \sim 156.25$ & 153.8 & 0.1529 & 4.6620 \\
$156.25 \sim 312.5$ & 219.1 & 0.4386 & 17.6503 \\
$312.5 \sim 625$ & 316.8 & 0.2489 & 5.5965 \\
$625 \sim 1250$ & 752.0 & 0.0745 & 0.1226 \\
$1250 \sim 2500$ & 2197.3 & 0.0291 & 0.0416 \\
$2500 \sim 5000$ & 2802.7 & 0.0438 & 0.0295 \\
\hline
\end{tabular}

TABLE 9: Dominant frequency, PPV, and energy proportion of each subfrequency band at measuring point 5 in the far zone.

\begin{tabular}{lccc}
\hline $\begin{array}{l}\text { Frequency band } \\
(\mathrm{Hz})\end{array}$ & $\begin{array}{c}\text { Dominant } \\
\text { frequency }(\mathrm{Hz})\end{array}$ & $\begin{array}{c}\text { PPV } \\
(\mathrm{cm} / \mathrm{s})\end{array}$ & $\begin{array}{c}\text { Energy } \\
\text { proportion }(\%)\end{array}$ \\
\hline $0 \sim 2.4415$ & 1.8 & 0.0030 & 0.2050 \\
$2.4415 \sim 4.8829$ & 3.7 & 0.0019 & 0.0180 \\
$4.8829 \sim 9.7657$ & 6.7 & 0.0019 & 0.0167 \\
$9.7657 \sim 19.5313$ & 14.6 & 0.0064 & 0.0730 \\
$19.5313 \sim 39.0625$ & 34.8 & 0.1199 & 12.3613 \\
$39.0625 \sim 78.125$ & 48.8 & 0.1981 & 14.5865 \\
$78.125 \sim 156.25$ & 121.5 & 0.2304 & 10.6664 \\
$156.25 \sim 312.5$ & 227.7 & 0.5570 & 51.9499 \\
$312.5 \sim 625$ & 330.8 & 0.2260 & 10.0215 \\
$625 \sim 1250$ & 912.5 & 0.0082 & 0.0485 \\
$1250 \sim 2500$ & 2269.9 & 0.0031 & 0.0337 \\
$2500 \sim 5000$ & 2730.1 & 0.0038 & 0.0195 \\
\hline
\end{tabular}

Step 1. Input the blasting vibration signal.

Step 2. Carry out multiresolution wavelet analysis on the blasting vibration signal.

Step 3. Obtain the energy proportion of each subfrequency band.

Step 4. Determine which zone the blasting vibration signal belongs to according to the following conditions. (1) When the energy proportion of high frequency band is higher than that of medium frequency band and low frequency band, the corresponding signal is divided into blasting vibration near zone. (2) When the energy proportion of medium frequency band is higher than that of high frequency band and low frequency band, the corresponding signal is divided into blasting vibration medium zone. (3) When the energy proportion of low frequency band is higher than that of high frequency band and medium frequency band, the corresponding signal is divided into blasting vibration far zone.

According to the zoning method of blasting vibration based on energy proportion, the frequency of signal 28 in Table 4 is mainly in the high frequency band, which is divided into blasting vibration near zone. The frequency of signal 57 in Table 5 is mainly in the medium frequency band, which is divided into blasting vibration medium zone. The frequency of signal 71 in Table 6 is mainly in the low frequency band, which is divided into blasting vibration far zone. The frequency of signal 10 in Table 7 is mainly in the medium frequency band, which is divided into blasting vibration medium zone. The frequency of signal 6 in Table 8 is mainly in the low frequency band, which is divided into blasting vibration far zone. The frequency of signal 5 in Table 9 is mainly in the high frequency band, which is divided into blasting vibration near zone.

\section{SVM Classification Models of Blasting Vibration Zoning Method}

Having enough samples with good typicality and high precision is an important condition for SVM modelling. 343 sets of representative effective signals were selected from a large number of measured blasting vibration signals, and the results by wavelet multiresolution analysis were taken as samples of SVM classification models. Table 10 shows the energy proportion results of 343 sets of measured blasting vibration signals after multiresolution wavelet analysis and the classification results by the 


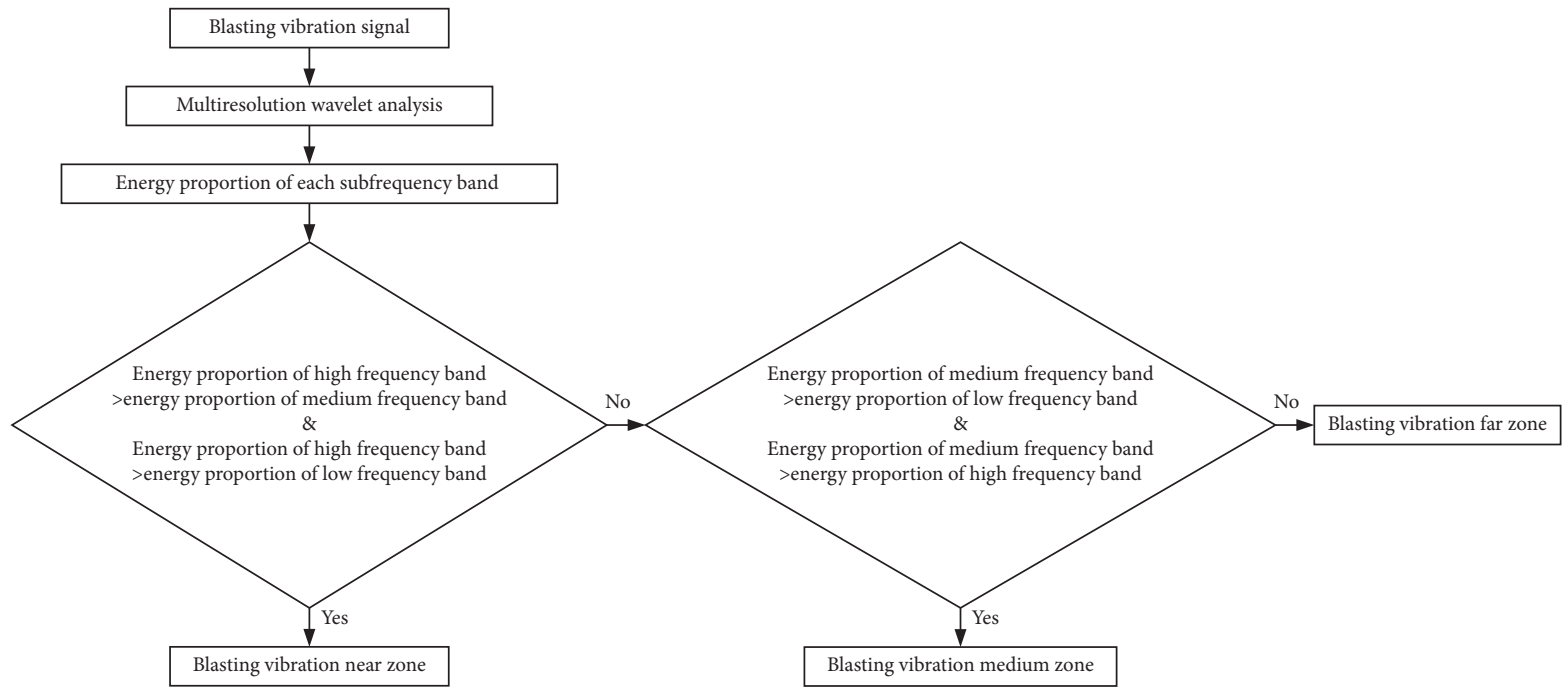

FIGURE 1: Flowchart of the zoning method of blasting vibration based on energy proportion.

blasting vibration zoning method proposed in Section 3.3. Labels 1 to 3 represent blasting vibration near, medium, and far zones, respectively.

Using simple random sampling method, 257 sets of samples (75\% of the total number of samples) in Table 10 were selected as training samples of SVM classification models and the SVM classification models were established. Among the 257 sets of training samples, there are 28 sets of samples in the near zone, 195 sets of samples in the medium zone, and 34 sets of samples in the far zone. The remaining 86 sets of samples ( $25 \%$ of the total number of samples) were taken as testing samples to verify the established models. Among the 86 sets of testing samples, there are 28 sets of samples in the near zone, 45 sets of samples in the medium zone, and 13 sets of samples in the far zone. As shown in Table 10, samples 1 to 257 are training samples, and samples 258 to 343 are testing samples.

In order to reduce the influence of the large difference between the parameters of the samples on the performance of prediction models, all sample data should be normalized according to the following formula before the training of SVM classification models [56]:

$$
X_{i j}=\frac{2\left(x_{i j}-x_{\min }(i)\right)}{x_{\max }(i)-x_{\min }(i)}-1,
$$

where $x_{i j}$ is the measured value of the $i$ th variable in the $j$ th sample and $x_{\max }(i)$ and $x_{\min }(i)$ are the maximum and minimum measured values of the $i$ th variables.

All the sample data in Table 10 were normalized to $[-1$, 1] by formula (15). After the classification of the models was completed, the classification results were also anti-normalized by using this formula.

Four types of kernel functions including LKF, PKF, RBF, and SKF were used to train SVM classification models, and different parameters were selected by the cross validation method for SVM classification models with any type of kernel function. Then, the optimal parameters were obtained for training models, and the blasting vibration zoning models based on energy proportion were established.

The accuracy of SVM classification models is evaluated by the following formula:

$$
\text { accuracy }=\frac{N_{c}}{N_{t}} \times 100 \%,
$$

where $N_{c}$ is number of samples correctly classified and $N_{t}$ is total number of samples.

The flowchart of SVM classification models of the proposed blasting vibration zoning method in this paper is shown in Figure 2. follows:

The detailed steps of SVM classification models are as

Step 1. The data are normalized and divided into training samples and testing samples.

Step 2. Initialize the parameters of SVM classification models, which include penalty coefficient and kernel function parameters.

Step 3. Optimize the penalty coefficient and kernel parameters. In SVM training, the penalty coefficient and kernel function parameters take discrete values in a certain range, and then the optimal values with the highest accuracy are obtained by the cross validation method.

Step 4. The optimal parameters obtained in Step 3 are used as the parameters of SVM classification models to classify the testing samples. If the end conditions are met, the SVM classification models and the classification accuracy are saved. Otherwise, return to Step 3.

Step 5. Output the SVM classification models with four kernel functions and their classification accuracy.

The parameters and accuracy of SVM classification models with four kernel functions based on the 343 sets of samples are shown in Table 11.

It can be seen from Table 11 that the average values of classification accuracy of SVM models with LKF, PKF, RBF, 
TABLE 10: Samples of SVM classification models.

\begin{tabular}{|c|c|c|c|c|c|c|c|c|c|c|c|c|c|}
\hline No. & PD1 & PD2 & PD3 & PD4 & PD5 & PD6 & PD7 & PD8 & PD9 & PD10 & PD11 & PD12 & Types \\
\hline 1 & 34.9899 & 0.0006 & 0.0053 & 0.0837 & 4.6407 & 55.7647 & 3.5752 & 0.7976 & 0.1192 & 0.0087 & 0.0086 & 0.0057 & 2 \\
\hline 2 & 0.4266 & 0.0057 & 0.0325 & 0.9285 & 5.0815 & 69.3728 & 22.8396 & 1.282 & 0.0199 & 0.0039 & 0.0038 & 0.0033 & 2 \\
\hline 3 & 10.1402 & 0.0009 & 0.0036 & 0.3908 & 10.61 & 69.7286 & 8.7192 & 0.3669 & 0.0357 & 0.0014 & 0.0015 & 0.0013 & 2 \\
\hline 4 & 49.9183 & 0.0017 & 0.0049 & 0.3082 & 6.5566 & 32.9399 & 9.4449 & 0.7546 & 0.0362 & 0.0134 & 0.0132 & 0.0081 & 3 \\
\hline 5 & 0.669 & 0.3257 & 0.3221 & 0.3979 & 0.9096 & 19.9203 & 49.1775 & 8.13 & 15.3682 & 2.0379 & 2.6942 & 0.0477 & 1 \\
\hline 6 & 5.3003 & 0.0229 & 0.4616 & 1.1009 & 2.7226 & 25.3614 & 10.2591 & 40.1265 & 14.2514 & 0.2683 & 0.1195 & 0.0056 & 1 \\
\hline 7 & 20.8464 & 0.4202 & 0.4336 & 0.4739 & 0.2685 & 0.8827 & 4.3216 & 15.2323 & 56.4006 & 0.4078 & 0.3068 & 0.0055 & 1 \\
\hline 8 & 0.2394 & 0.0056 & 0.1217 & 0.8419 & 16.961 & 77.2803 & 4.4831 & 0.0613 & 0.0025 & 0.001 & 0.0011 & 0.0011 & 2 \\
\hline 9 & 3.552 & 1.4435 & 0.9823 & 2.4488 & 20.0208 & 34.9933 & 32.1278 & 2.9461 & 0.8424 & 0.3046 & 0.123 & 0.2153 & 2 \\
\hline 10 & 1.2521 & 0.8527 & 0.6572 & 0.3043 & 0.7247 & 2.9896 & 35.9766 & 33.3059 & 19.3597 & 3.0272 & 1.5348 & 0.0152 & 1 \\
\hline 11 & 19.5308 & 0.22 & 0.3121 & 0.1818 & 0.2147 & 0.7938 & 0.8867 & 34.9105 & 41.4989 & 0.9115 & 0.5299 & 0.0093 & 1 \\
\hline 12 & 0.0423 & 0.002 & 0.0051 & 0.6367 & 4.7189 & 84.4154 & 10.1338 & 0.0437 & 0.0006 & 0.0005 & 0.0005 & 0.0005 & 2 \\
\hline 13 & 9.5117 & 0.0009 & 0.0031 & 0.9062 & 70.0943 & 18.7993 & 0.6401 & 0.0257 & 0.0064 & 0.0042 & 0.0042 & 0.0038 & 2 \\
\hline 14 & 9.9489 & 0.0012 & 0.0978 & 0.6183 & 28.6976 & 58.4203 & 2.1463 & 0.0408 & 0.0102 & 0.0073 & 0.0059 & 0.0053 & 2 \\
\hline 15 & 0.2728 & 0.0038 & 0.1004 & 0.5473 & 13.5466 & 80.2582 & 5.2088 & 0.0574 & 0.0017 & 0.0009 & 0.001 & 0.001 & 2 \\
\hline$\ldots$ & $\ldots$ & $\ldots$ & $\ldots$ & $\ldots$ & $\ldots$ & $\ldots$ & ... & $\ldots$ & $\ldots$ & $\ldots$ & ... & $\ldots$ & ... \\
\hline 329 & 45.168 & 0.0029 & 0.112 & 0.3264 & 15.502 & 20.2044 & 16.43 & 2.0677 & 0.1224 & 0.0257 & 0.0167 & 0.0133 & 3 \\
\hline 330 & 1.2114 & 0.017 & 0.0515 & 0.3248 & 10.7671 & 45.9 & 40. & 1.5904 & 0.0439 & 0.0175 & 0.014 & 0.0108 & 2 \\
\hline 331 & 65.8766 & 0.0094 & 0.0163 & 0.1364 & 6.6911 & 13.4421 & 11.9669 & 1.2144 & 0.5079 & 0.0615 & 0.0417 & 0.0358 & 3 \\
\hline 332 & 0.2774 & 0.0207 & 0.0791 & 0.1799 & 8.379 & 67.579 & 22.7869 & 0.6862 & 0.005 & 0.0025 & 0.002 & 0.0022 & 2 \\
\hline 333 & 13.1182 & 0.0008 & 0.0144 & 0.0923 & 16.8056 & 59.9594 & & & & 0.0095 & 0.0 & & 2 \\
\hline 334 & 0.4644 & 0.0267 & 0.1362 & 0.9169 & 5.8446 & 72.4972 & 19.0 & 1.0113 & 0.0067 & 0.0036 & 0.0032 & 0.0034 & 2 \\
\hline 335 & 23.3669 & 0.001 & 0.0079 & 1.3397 & 21.1059 & 34.2772 & 17.7088 & 2.0573 & 0.0974 & 0.0166 & 0.0119 & 0.0093 & 2 \\
\hline 336 & 0.9944 & 0.0034 & 0.1349 & 1.4361 & 8.5149 & 51.1464 & 36.9286 & 0.7893 & 0.0194 & 0.0119 & 0.0123 & 0.0083 & 2 \\
\hline 337 & 12.0654 & 0.0007 & 0.0019 & 0.2775 & 11.6415 & 71.6 & 4.0703 & 0.2 & 0.021 & 0.0014 & 0.0 & 0.0013 & 2 \\
\hline 338 & 76.8729 & 0.0027 & 0.0203 & 0.1738 & 4.5146 & 12.6639 & 5.4112 & 0.2615 & 0.0361 & 0.0157 & 0.0163 & 0.011 & 3 \\
\hline 339 & 1.7324 & 0.0013 & 0.0571 & 0.3428 & 1.4073 & 84.1498 & 10.6934 & 1.519 & 0.0651 & 0.0111 & 0.0112 & 0.0095 & 2 \\
\hline 340 & 26.6758 & 0.0012 & 0.0165 & 0.2755 & 18.7241 & 46.8901 & 6.8235 & 0.5219 & 0.0629 & 0.0027 & 0.0031 & 0.0027 & 2 \\
\hline 341 & 85.1141 & 0.0011 & 0.0043 & 0.0881 & 2.8739 & 7.9185 & 3.4547 & 0.406 & 0.0724 & 0.0264 & 0.0252 & 0.0153 & 3 \\
\hline 342 & 0.2431 & 0.0013 & 0.0351 & 0.204 & 5.1819 & 83.2178 & 9.4493 & 1.6365 & 0.0255 & 0.0018 & 0.002 & 0.0017 & 2 \\
\hline 343 & 8.4562 & 0.0004 & 0.003 & 0.0743 & 7.5993 & 74.9625 & 8.3907 & 0.4444 & 0.0654 & 0.0014 & 0.0013 & 0.0012 & 2 \\
\hline
\end{tabular}

Note. PD1 PD12, respectively, represent frequency bands of $0 \sim 2.441 \mathrm{~Hz}, 2.441 \sim 4.882 \mathrm{~Hz}, 4.882 \sim 9.765 \mathrm{~Hz}, 9.765 \sim 19.531 \mathrm{~Hz}, 19.531 \sim 39.062 \mathrm{~Hz}$, $39.062 \sim 78.125 \mathrm{~Hz}, 78.125 \sim 156.25 \mathrm{~Hz}, 156.25 \sim 312.5 \mathrm{~Hz}, 312.5 \sim 625 \mathrm{~Hz}, 625 \sim 1250 \mathrm{~Hz}, 1250 \sim 2500 \mathrm{~Hz}$, and $2500 \sim 5000 \mathrm{~Hz}$.

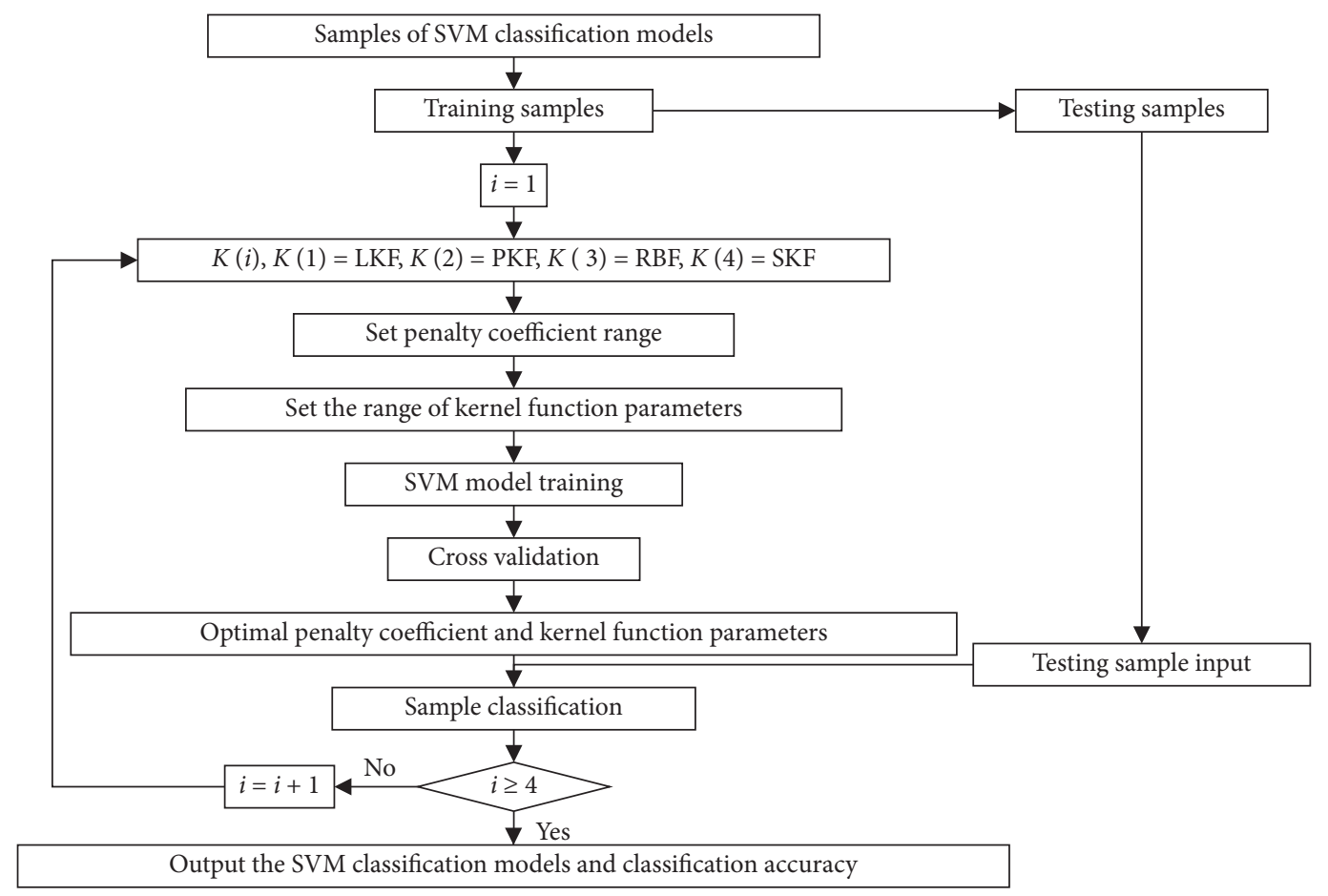

FIGURE 2: Flowchart of SVM classification models. 
TABLE 11: Parameters and prediction accuracy of SVM classification models with different kernel functions.

\begin{tabular}{|c|c|c|c|c|c|c|c|c|c|}
\hline \multirow[b]{2}{*}{ No. } & \multicolumn{3}{|c|}{ Kernel functions } & \multicolumn{6}{|c|}{ SVM classification models } \\
\hline & Types & Parameters & Values & $c$ & Iterations & $\begin{array}{l}\text { Number of support } \\
\text { vectors }\end{array}$ & $\begin{array}{l}\text { Accuracy of training } \\
\text { samples }\end{array}$ & $\begin{array}{c}\text { Accuracy of } \\
\text { testing } \\
\text { samples }\end{array}$ & $\begin{array}{l}\text { Average values of } \\
\text { prediction accuracy }\end{array}$ \\
\hline 1 & \multirow{10}{*}{ LKF } & \multirow{10}{*}{-} & \multirow{10}{*}{-} & 4.5948 & 35 & 35 & 99.2218 & 97.6744 & \multirow{10}{*}{97.7907} \\
\hline 2 & & & & 48.5029 & 92 & 20 & 100 & 96.5116 & \\
\hline 3 & & & & 8 & 40 & 29 & 99.2218 & 97.6744 & \\
\hline 4 & & & & 13.9288 & 92 & 26 & 100 & 97.6744 & \\
\hline 5 & & & & 6.9644 & 41 & 30 & 99.2218 & 97.6744 & \\
\hline 6 & & & & 24.2515 & 92 & 22 & 100 & 98.8372 & \\
\hline 7 & & & & 21.1121 & 92 & 23 & 100 & 98.8372 & \\
\hline 8 & & & & 10.5561 & 76 & 29 & 99.6109 & 97.6744 & \\
\hline 9 & & & & 4 & 35 & 35 & 99.2218 & 97.6744 & \\
\hline 10 & & & & 6.0629 & 46 & 31 & 99.2218 & 97.6744 & \\
\hline 11 & \multirow{10}{*}{ PKF } & \multirow{10}{*}{$d$} & 1 & 0.0045 & 28 & 114 & 75.8755 & 53.3256 & \multirow{10}{*}{92.1930} \\
\hline 12 & & & 2 & 4 & 82 & 21 & 100 & 96.5116 & \\
\hline 13 & & & 3 & 27.8576 & 48 & 21 & 100 & 97.6744 & \\
\hline 14 & & & 4 & 32 & 24 & 22 & 100 & 97.6744 & \\
\hline 15 & & & 5 & 42.2243 & 24 & 22 & 100 & 96.5116 & \\
\hline 16 & & & 6 & 36.7583 & 22 & 23 & 100 & 95.3488 & \\
\hline 17 & & & 7 & 32 & 21 & 23 & 100 & 95.3488 & \\
\hline 18 & & & 8 & 42.2243 & 20 & 25 & 100 & 96.5116 & \\
\hline 19 & & & 9 & 42.2243 & 17 & 26 & 100 & 96.5116 & \\
\hline 20 & & & 10 & 55.7152 & 17 & 28 & 100 & 96.5116 & \\
\hline 21 & \multirow{10}{*}{$\mathrm{RBF}$} & \multirow{10}{*}{$g$} & 0.1649 & 27.8576 & 54 & 33 & 99.2218 & 98.8372 & \multirow{10}{*}{98.7209} \\
\hline 22 & & & 0.0625 & 73.5167 & 35 & 30 & 99.2218 & 98.8372 & \\
\hline 23 & & & 0.0947 & 24.2515 & 31 & 35 & 99.2218 & 98.8372 & \\
\hline 24 & & & 0.1250 & 21.1121 & 37 & 36 & 99.2218 & 98.8372 & \\
\hline 25 & & & 0.1088 & 32 & 32 & 32 & 99.2218 & 98.8372 & \\
\hline 26 & & & 0.1436 & 32 & 47 & 33 & 99.2218 & 98.8372 & \\
\hline 27 & & & 0.1250 & 27.8576 & 33 & 34 & 99.2218 & 98.8372 & \\
\hline 28 & & & 0.1436 & 48.5029 & 137 & 32 & 100 & 98.8372 & \\
\hline 29 & & & 0.1649 & 21.1121 & 39 & 34 & 99.6109 & 98.8372 & \\
\hline 30 & & & 0.0136 & 168.8970 & 34 & 35 & 99.2218 & 97.6744 & \\
\hline 31 & \multirow{10}{*}{ SKF } & \multirow{10}{*}{$k$} & 0.0825 & 84.4485 & 35 & 35 & 99.2218 & 97.6744 & \multirow{10}{*}{97.7907} \\
\hline 32 & & & 0.0625 & 97.0059 & 25 & 35 & 99.2218 & 97.6744 & \\
\hline 33 & & & 0.0947 & 445.7219 & 75 & 24 & 100 & 98.8372 & \\
\hline 34 & & & 0.0718 & 168.8970 & 43 & 28 & 99.2218 & 97.6744 & \\
\hline 35 & & & 0.0825 & 168.8970 & 60 & 29 & 99.2218 & 97.6744 & \\
\hline 36 & & & 0.0421 & 675.5881 & 68 & 22 & 100 & 98.8372 & \\
\hline 37 & & & 0.0825 & 388.0234 & 80 & 24 & 100 & 97.6744 & \\
\hline 38 & & & 0.0947 & 222.8609 & 54 & 26 & 99.6109 & 97.6744 & \\
\hline 39 & & & 0.1895 & 36.7583 & 48 & 51 & 99.2218 & 96.5116 & \\
\hline 40 & & & 0.0272 & 168.8970 & 25 & 35 & 99.2218 & 97.6744 & \\
\hline
\end{tabular}

and SKF are $97.7907 \%, 92.1930 \%, 98.7209 \%$, and $97.7907 \%$, respectively, among which the accuracy of SVM model with $\mathrm{RBF}$ is the highest. Based on the SVM classification models with RBF of Nos. 21 to 27, the accuracy to classify the training samples is $99.2218 \%$ and the accuracy to classify the testing samples is $98.8372 \%$. Based on the SVM classification model with RBF of No. 28, the accuracy to classify the training samples is $100 \%$ and the accuracy to classify the testing samples is $98.8372 \%$. Based on the SVM classification model with RBF of No. 29, the accuracy to classify the training samples is $99.6109 \%$ and the accuracy to classify the testing samples is $98.8372 \%$. Based on the SVM classification model with RBF of No. 30, the accuracy to classify the training samples is $99.2218 \%$ and the accuracy to classify the testing samples is $97.6744 \%$. All the SVM classification models with $\mathrm{RBF}$ of different parameters selected by the cross validation method show good prediction effect in blasting vibration zoning prediction, and the prediction accuracy of all the SVM models with RBF is more than $97.5 \%$ without overfitting. The classification results show that the zoning method of blasting vibration based on energy proportion proposed in this paper has high feasibility, flexibility, and reliability, and the classification algorithm of SVM models with RBF has high accuracy in blasting vibration zoning prediction.

The prediction results of testing samples by SVM classification models with RBF of Nos. 21 to 29 in Table 11 are shown in Figure 3, and the prediction results of testing samples by SVM classification model with RBF of No. 30 in Table 11 are shown in Figure 4 . It can be found that all the 


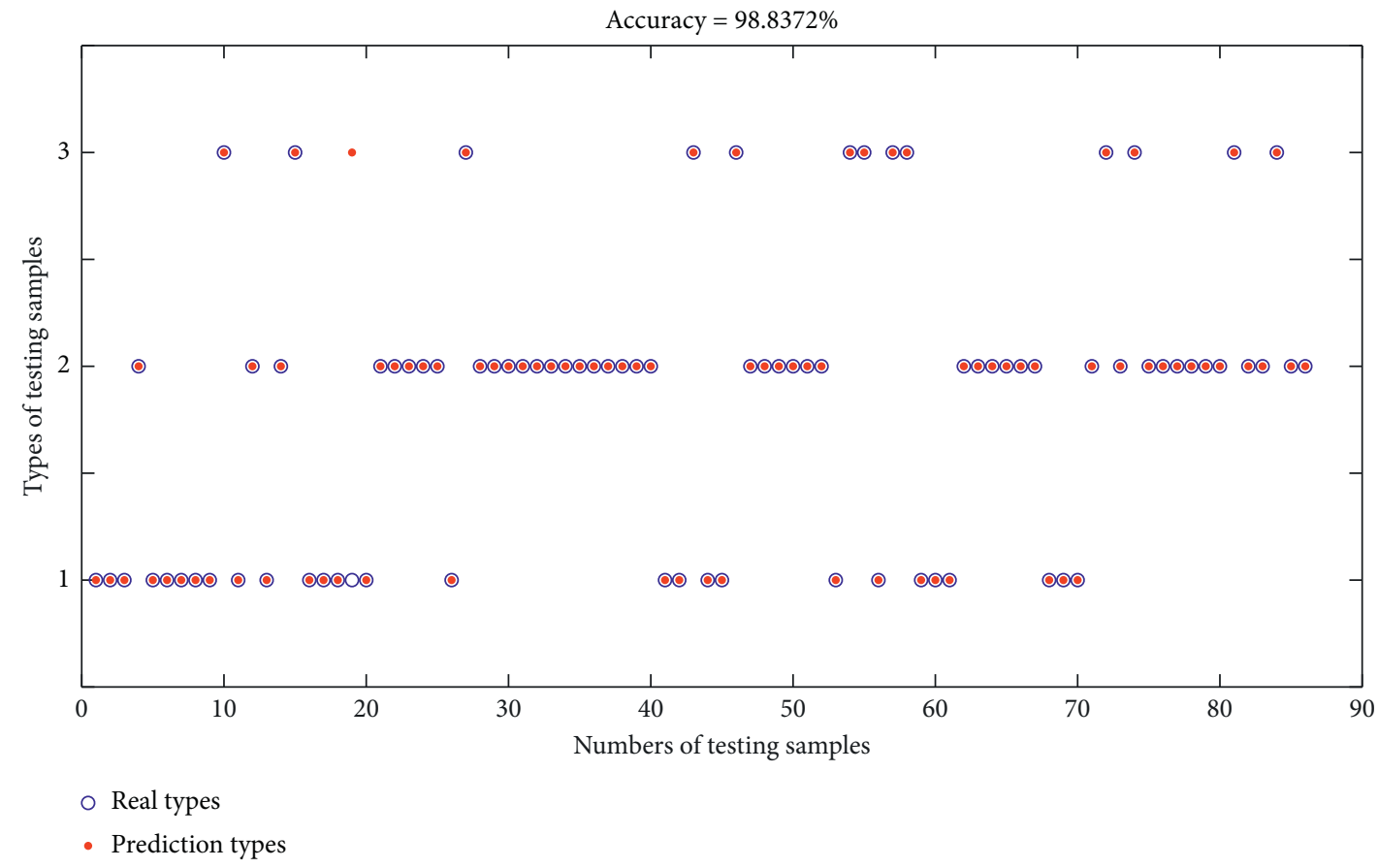

Figure 3: Prediction results of testing samples by SVM classification models of Nos. 21 to 29.

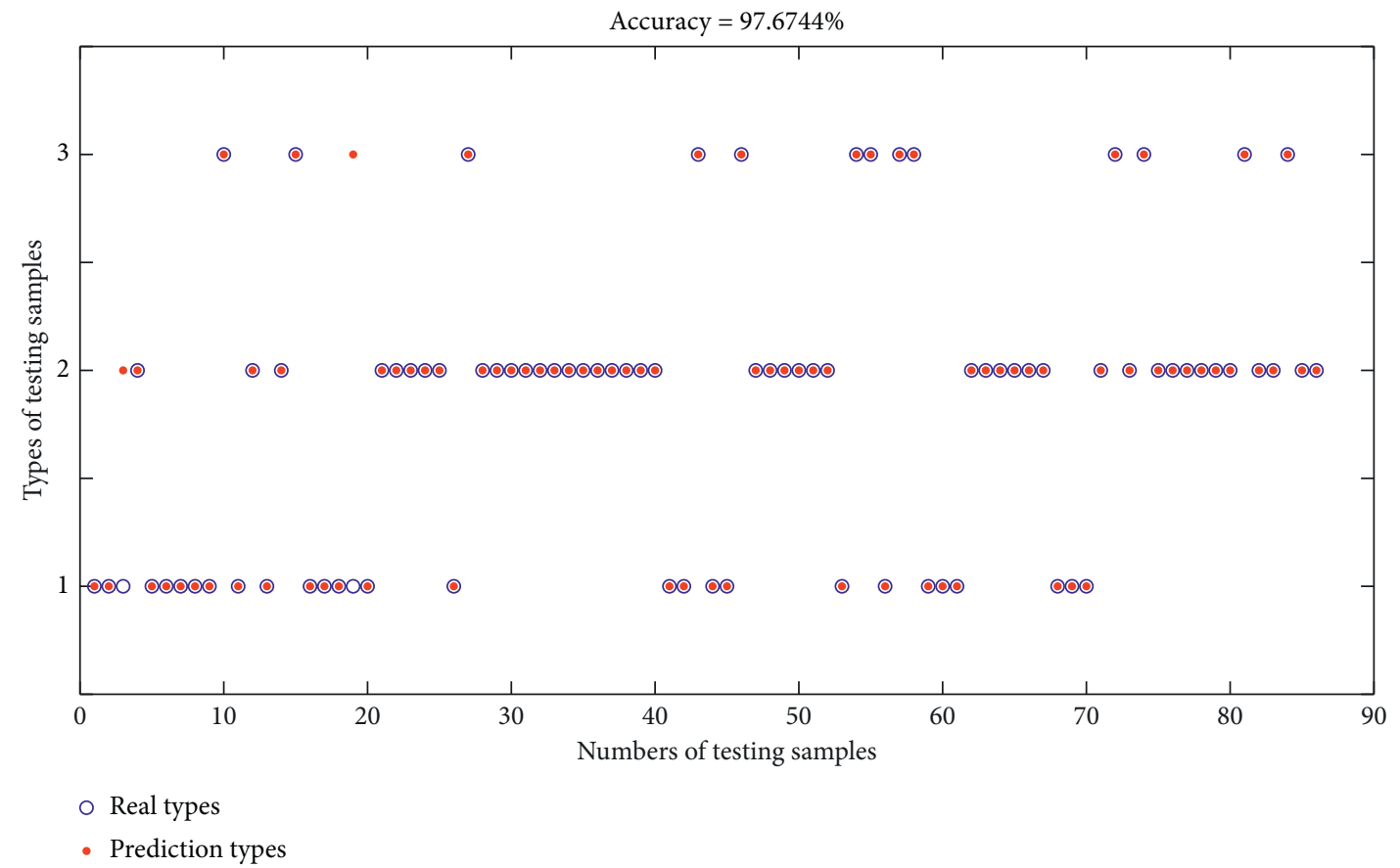

FIgURE 4: Prediction results of testing samples by SVM classification model of No. 30.

SVM classification models with RBF of Nos. 21 to 30 of different parameters in Table 11 classify sample 19 (corresponding to sample 276 in Table 10) incorrectly, and the SVM classification model with RBF of No. 30 classifies sample 3 (corresponding to sample 260 in Table 10) incorrectly. The energy proportions of sample 19 in frequency band $0 \sim 2.4415 \mathrm{~Hz}(43.6350 \%)$ and sample 3 in frequency band $39.062 \sim 78.125 \mathrm{~Hz}(28.3373 \%)$ are both more than $10 \%$. It is considered that the energy proportion of the two frequency bands cannot be ignored according to reference [57]. Therefore, the SVM classification models with RBF cannot identify and classify samples 19 and 3 correctly, which reflects the influence of sample typicality on the prediction effect of the SVM classification algorithm. 


\section{Conclusions}

The automatic identification and classification of blasting vibration signals in different zones is a complex subject, which is a powerful means to study the detailed characteristics for blasting vibration signals. Because of not considering the specific influences of geological and topographical conditions, the existing zoning methods for blasting vibration have some limitations. In this study, a new blasting vibration zoning method based on energy proportion was proposed. The energy proportion characteristics of each subfrequency band were extracted by multiresolution wavelet analysis, and the blasting vibration near, medium, and far zones were divided corresponding to the dominant energy proportion of high, medium, and low frequency bands.

Based on the proposed zoning method, 257 sets of measured blasting vibration signals were selected as training samples and 86 sets were selected as testing samples to train and test the SVM classification models with four different kernel functions of LKF, PKF, RBF, and SKF. All the SVM classification models with RBF of different parameters selected by the cross validation method show good prediction effect in blasting vibration zoning prediction, and the prediction accuracy of all the SVM models with RBF is more than $97.5 \%$ without overfitting. It is proved that the zoning method of blasting vibration based on energy proportion proposed in this paper has high feasibility, flexibility, and reliability, and the SVM classification models with RBF have high prediction accuracy.

However, there may be some mistakes in the classification of atypical signals when the number of signal samples in some blasting vibration zones is relatively small [58] and the energy proportion of signals in other frequency band is more than $10 \%$. As in this paper, because there are more samples in the medium zone than those in the near and far zones, SVM classification models are very good for the classification of atypical signals in the medium zone, and even the accuracy is $100 \%$. Therefore, in the future work, we can increase the number of signal samples in the near and far zones to enhance the identification ability of SVM classification models for atypical signals and further improve the classification accuracy.

The frequency bands of the near, medium, and far zones by the proposed zoning method of blasting vibration based on energy proportion are basically in accordance with the frequency bands of blasting vibration safety standard in "blasting safety regulations" [59]: (0, $10),(10,50),(50,100)$. Therefore, the zoning method can not only provide theoretical guidance for studying the specific characteristics of blasting vibration signals in each vibration zone but also provide an important basis for the prediction, control, and safety standards for blasting vibration.

\section{Data Availability}

The data used to support the findings of this study are included within the article.

\section{Conflicts of Interest}

The authors declare that there are no conflicts of interest regarding the publication of this paper.

\section{Acknowledgments}

This study was supported by the National Natural Science Foundation of China (nos. 51874123 and 51504082) and the Open Project Foundation of Fujian Research Center for Tunneling and Urban Underground Space Engineering (no. 16FTUE01).

\section{References}

[1] M. Khandelwal and M. Saadat, "A dimensional analysis approach to study blast-induced ground vibration," Rock Mechanics and Rock Engineering, vol. 48, no. 4, pp. 727-735, 2015.

[2] A. Sołtys, M. Twardosz, and J. Winzer, "Control and documentation studies of the impact of blasting on buildings in the surroundings of open pit mines," Journal of Sustainable Mining, vol. 16, no. 4, pp. 179-188, 2017.

[3] T. Wang, Z. Song, J. Yang et al., "Experimental research on dynamic response of red sandstone soil under impact loads," Geomechanics and Engineering, vol. 17, no. 4, pp. 393-403, 2019.

[4] M. Monjezi, M. Baghestani, R. Shirani Faradonbeh et al., "Modification and prediction of blast-induced ground vibrations based on both empirical and computational techniques," Engineering with Computers, vol. 32, no. 2, pp. 717-728, 2016.

[5] G. Liu, "Prediction of blasting vibration velocity for largesection tunnel blasting," Blasting, vol. 36, no. 3, pp. 129-136, 2019.

[6] H. Aawal and A. K. Mishra, "Modified scaled distance regression analysis approach for prediction of blast-induced ground vibration in multi-hole blasting," Journal of Rock Mechanics and Geotechnical Engineering, vol. 11, no. 1, pp. 202-207, 2019.

[7] V. K. Himanshu, M. P. Roy, A. K. Mishra et al., "Multivariate statistical analysis approach for prediction of blast-induced ground vibration," Arabian Journal of Geosciences, vol. 11, no. 16, 11 pages, Article ID 460, 2018.

[8] M. I. Matidza, Z. Jianhua, H. Gang, and A. D. Mwangi, "Assessment of blast-induced ground vibration at jinduicheng molybdenum open pit mine," Natural Resources Research, vol. 29, no. 2, pp. 831-841, 2020.

[9] M. Monjezi, M. Ghafurikalajahi, and A. Bahrami, "Prediction of blast-induced ground vibration using artificial neural networks," Tunnelling and Underground Space Technology, vol. 26, no. 1, pp. 46-50, 2011.

[10] M. Hasanipanah, M. Monjezi, A. Shahnazar, D. Jahed Armaghani, and A. Farazmand, "Feasibility of indirect determination of blast induced ground vibration based on support vector machine," Measurement, vol. 75, pp. 289-297, 2015.

[11] B. Mei, X. Wang, and R. Yang, "Prediction of blasting vibration intensity based on adaboost-SVM combination algorithm," Journal of Vibration and Shock, vol. 38, no. 18, pp. 231-235, 2019.

[12] Q. Yuan, S. Zhai, L. Wu, P. Chen, Y. Zhou, and Q. Zuo, "Blasting vibration velocity prediction based on least squares 
support vector machine with particle swarm optimization algorithm," Geosystem Engineering, vol. 22, no. 5, pp. 279-288, 2019.

[13] M. Hasanipanah, R. S. Faradonbeh, H. B. Amnieh, D. J. Armaghani, and M. Monjezi, "Forecasting blastinduced ground vibration developing a CART model," Engineering with Computers, vol. 33, no. 2, pp. 307-316, 2017.

[14] M. Hasanipanah, H. Bakhshandeh Amnieh, H. Khamesi, D. Jahed Armaghani, S. Bagheri Golzar, and A. Shahnazar, "Prediction of an environmental issue of mine blasting: an imperialistic competitive algorithm-based fuzzy system," International Journal of Environmental Science and Technology, vol. 15, no. 3, pp. 551-560, 2018.

[15] E. Tian, J. Zhang, M. Soltani Tehrani, A. Surendar, and A. Z. Ibatova, "Development of GA-based models for simulating the ground vibration in mine blasting," Engineering with Computers, vol. 35, no. 3, pp. 849-855, 2019.

[16] M. Hasanipanah, S. B. Golzar, I. A. Larki, M. Y. Maryaki, and T. Ghahremanians, "Estimation of blast-induced ground vibration through a soft computing framework," Engineering with Computers, vol. 33, no. 4, pp. 951-959, 2017.

[17] H. Eskandar, E. Heydari, M. Hasanipanah, M. Jalil Masir, and A. Mahmodi Derakhsh, "Feasibility of particle swarm optimization and multiple regression for the prediction of an environmental issue of mine blasting," Engineering Computations, vol. 35, no. 1, pp. 363-376, 2018.

[18] X. Bui, P. Jaroonpattanapong, H. Nguyen et al., "A novel hybrid model for predicting blast-induced ground vibration based on $k$-nearest neighbors and particle swarm optimization," ScientificReports, vol. 9, no. 1, 14 pages, Article ID 13971, 2019.

[19] H. Wei, J. Chen, J. Zhu, X. Yang, and H. Chu, "A novel algorithm of nested-ELM for predicting blasting vibration," Engineering with Computers, 2020.

[20] T. Guo, X. Fang, Q. Xie et al., "Application of FSWT in accurate extraction of time-frequency features for blasting vibration signals," Journal of Vibration and Shock, vol. 32, no. 22, pp. 73-78, 2013.

[21] J. Zhou, W. Lu, L. Zhang et al., "Attenuation of vibration frequency during propagation of blasting seismic wave," Chinese Journal of Rock Mechanics and Engineering, vol. 33, no. 11, pp. 2171-2178, 2014.

[22] Y. Peng, Y. Su, L. Wu et al., "Study on the attenuation characteristics of seismic wave energy induced by underwater drilling and blasting," Shock and Vibration, vol. 2019, Article ID 4367698, 13 pages, 2019.

[23] Q. Gao, W. Lu, Z. Leng et al., "Effect of initiation location within blast hole on blast vibration field and its mechanism," Shock and Vibration, vol. 2019, Article ID 5386014, 18 pages, 2019.

[24] W. Lu, J. Zhou, M. Chen et al., "Study on attenuation formula of dominant frequency of blasting vibration," Engineering Blasting, vol. 21, no. 6, 6 pages, 2015.

[25] H. Li, X. Li, J. Li, X. Xia, and X. Wang, "Application of coupled analysis methods for prediction of blast-induced dominant vibration frequency," Earthquake Engineering and Engineering Vibration, vol. 15, no. 1, pp. 153-162, 2016.

[26] D. Liu, W. Lu, Y. Liu et al., "Analysis of the main factors influencing the dominant frequency of blast vibration," Shock and Vibration, vol. 2019, Article ID 8480905, 17 pages, 2019.

[27] Y. Gou, X. Shi, J. Zhou et al., "Attenuation assessment of blastinduced vibrations derived from an underground mine,"
International Journal of Rock Mechanics and Mining Sciences, vol. 127, p. 13, Article ID 104220, 2020.

[28] B. Li, "Vibration effect of longhole blasting in open pit mining," Mining Research and Development, vol. 9, no. 4, pp. 84-94, 1989.

[29] H. Pang and S. Chen, "Variation law of blasting seismic wave's propagation in elastic media," Journal of Vibration and Shock, vol. 28, no. 3, pp. 105-107, 2009.

[30] Z. Zhang, C. Lin, Z. Huang et al., "Prediction of blasting vibration of area near tunnel blasting source," Explosion and Shock Waves, vol. 34, no. 3, pp. 367-372, 2014.

[31] D. Meng, Study on Construction Blasting Vibration Influence and Partition in Railway Interchange Tunnel, Central South University, Changsha, China, 2014.

[32] H. Wang, Study on Blasting Vibration Propagation and Partition in Super Large Section Small Spacing Tunnel, Southwest Jiaotong University, Chengdu, China, 2019.

[33] Z. Du, R. Zhang, and H. Chen, "Characteristic signal extracted from a continuous time signal on the aspect of frequency domain," Chinese Physics B, vol. 28, Article ID 090502, 7 pages, 2019.

[34] W. Deng, H. Liu, J. Xu, H. Zhao, and Y. Song, "An improved quantum-inspired differential evolution algorithm for deep belief network," IEEE Transactions on Instrumentation and Measurement, vol. 69, no. 10, pp. 7319-7327, 2020.

[35] Y. Song, D. Wu, W. Deng et al., "MPPCEDE: multi-population parallel co-evolutionary differential evolution for parameter optimization," Energy Conversion and Management, vol. 228, Article ID 113661, 19 pages, 2021.

[36] Y. Song, D. Wu, A. Mohamed et al., "Enhanced success history adaptive DE for parameter optimization of photovoltaic models," Complexity, vol. 2021, Article ID 6660115, 2021.

[37] "Classification algorithm SVM (support vector machine)," 2020, https://blog.csdn.net/dcrmg/article/details/53000150.

[38] X. Wang, S. Yan, D. Li et al., "Pedestrian navigation nerospeed correction method based on support vector machine classification decision," Science Technology and Engineering, vol. 19, no. 1, pp. 159-165, 2019.

[39] S. Solikin, H. Manik, S. Pujiyati et al., "Support vector machine classification method for predicting Jakarta Bay bottom sediment type using multibeam echosounder data," Pertanika Journal of Science and Technology, vol. 48, no. 2, pp. 477-491, 2020.

[40] L. Tang, M. Zhang, and L. Wen, "Support vector machine classification of seismic events in the Tianshan orogenic belt," Journal of Geophysical Research: Solid Earth, vol. 125, no. 1, 2020.

[41] C. Yang and J. Wu, "Discriminating blasting vibration and rock fracture micro-seismic signal based on wavelet packet analysis and SVM," Bulletin of Science and Technology, vol. 35, no. 1, pp. 19-23, 2019.

[42] V. Vapnik, Statistical Learning Theory, John Wiley \& Sons Inc, New York, NY, USA, 1998.

[43] V. Vapnik, The Nature of Statistical Learning Theory, Springer-Verlag, New York, NY, USA, 2000.

[44] N. Cristianini and J. Shawa-Taylor, An Introduction of Support Vector Machines and Other Kernel-Based Learning Methods, Cambridge University Press, Cambridge, UK, 2000.

[45] C. J. C. Burges, "A tutorial on support vector machines for pattern recognition," Data Mining and Knowledge Discovery, vol. 2, no. 2, pp. 121-167, 1998.

[46] Y. Chen, X. Yu, X. Gao et al., "A new method for non-linear classify and non-linear regression I: introduction to support 
vector machine," Journal of Applied Meteorological Science, vol. 15, no. 3, pp. 345-354, 2004.

[47] W. Lu, N. Chen, C. Ye et al., "Introduction to the algorithm of support vector machine and the software Chem SVM," Computers and Applied Chemistry, vol. 19, no. 6, pp. 697-702, 2002.

[48] L. Yu, 30 Cases Analysis of MATLAB Intelligent Algorithm, Beijing University Press, Beijing, China, 2011.

[49] C. Cortes and V. Vapnik, "Support-vector networks," Machine Learning, vol. 20, no. 3, pp. 273-297, 1995.

[50] I. Daubechies, "Orthonormal bases of compactly supported wavelets," Communications on Pure and Applied Mathematics, vol. 41, no. 7, pp. 909-996, 1988.

[51] Y. Sun and D. Liu, "Time-frequency analysis and wavelet transform and their applications," Engineering Journal of Wuhan University, vol. 36, no. 2, pp. 103-106, 2003.

[52] S. Li, "Wavelet transform and applications," Progress in Geophysics, vol. 7, no. 2, pp. 45-53, 1992.

[53] J. Wang, M. Wu, and Y. Sun, "Wavelet transform and applications," Journal of Henan Normal University, vol. 30, no. 2, pp. 86-89, 2002.

[54] S. Chen, H. Wei, and R. Du, "Multi-resolution wavelet analysis of blasting vibration signals," Rock and Soil Mechanics, vol. 30, no. s1, pp. 135-139, 2009.

[55] T. Liu, X. Zeng, and J. Zeng, Introduction to Practical Wavelet Analysis, National Defense Industry Press, Beijing, China, 2006.

[56] E. M. Golafshani and A. Ashour, "A feasibility study of BBP for predicting shear capacity of FRP reinforced concrete beams without stirrups," Advances in Engineering Software, vol. 97, pp. 29-39, 2016.

[57] Intellectual Property Publishing House, CN201811310006.3 Method for Constructing Blasting Seismic Wave Model Equivalent Based on Building Earthquake Response, Intellectual Property Publishing House, Beijing, China, 2019.

[58] J. Zhou, E. Li, H. Wei et al., "Random forests and cubist algorithms for predicting shear strengths of rockfill materials," Applied Sciences, vol. 9, no. 8, 16 pages, Article ID 9081621, 2019.

[59] Standardization Administration of China, Chinese National Standard GB6722-2014: Safety Regulation for Blasting, Standardization Administration of China, Beijing, China, 2014. 